\title{
Characterisation of a SAT-1 outbreak of foot-and- mouth disease in captive African buffalo (Syncerus caffer): Clinical symptoms, genetic characterisation and phylogenetic comparison of outbreak isolates
}

\author{
W. Vosloo ${ }^{\text {a, b }}$, L.-M. de Klerk ${ }^{c}$, C.I. Boshoff ${ }^{\text {a, d }}$, B. Botha ${ }^{a}$, R.M. Dwarka ${ }^{a}$, D. Keet ${ }^{e}$ \\ and D.T. Haydon
}

${ }^{\mathrm{a} O n d e r s t e p o o r t ~ V e t e r i n a r y ~ I n s t i t u t e, ~ P r i v a t e ~ B a g ~ X 05, ~ O n d e r s t e p o o r t ~ 0110, ~ S o u t h ~ A f r i c a ~}$

${ }^{\mathrm{b}}$ Department of Veterinary Tropical Diseases, University of Pretoria, Pretoria 0002, South Africa

${ }^{c}$ Veterinary Wildlife Services, Kruger National Park, P.O. Box 156, Skukuza 1350, South Africa

${ }^{\mathrm{d}}$ Department of Biomedical Sciences, Tshwane niversity of Technology, Private Bag X680, Pretoria 0001, South Africa

${ }^{\text {e}}$ Office of the State Veterinarian, Kruger National Park, PO Box 12, Skukuza 1350, South Africa

${ }_{\mathrm{f}}^{\mathrm{Division}}$ of Environmental and Evolutionary Biology, University of Glasgow, Glasgow G12 8QQ, UK

\section{Abstract}

African buffalo (Syncerus caffer) play an important role in the maintenance of the SAT types of foot-and-mouth disease (FMD) in southern Africa. These long-term carriers mostly become sub-clinically infected, maintaining the disease and posing a threat to other susceptible wildlife and domestic species. During an unrelated bovine tuberculosis experiment using captive buffalo in the Kruger National Park (KNP), an outbreak of SAT-1 occurred and was further investigated. The clinical signs were recorded and all animals demonstrated significant weight loss and lymphopenia that lasted 100 days. In 
addition, the mean cell volume and mean cell haemoglobin values were significantly higher than before the outbreak started. Virus was isolated from several buffalo over a period of 167 days post infection and the molecular clock estimated to be $3 \times 10^{-5}$ nucleotide substitutions per site per day. Seven amino acid changes occurred of which four occurred in hypervariable regions previously described for SAT-1. The genetic relationship of the outbreak virus was compared to buffalo viruses previously obtained from the KNP but the phylogeny was largely unresolved, therefore the relationship of this outbreak strain to others isolated from the KNP remains unclear.

\section{Article Outline}

1. Introduction

2. Materials and methods

2.1. Study group and description of the outbreak

2.2. Viruses included in the study

2.3. Haematology

2.4. Collection of clinical material

2.5. Virus isolation on cell cultures

2.6. RNA extraction and cDNA synthesis

2.7. Polymerase chain reaction

2.8. PCR product purification and nucleotide sequencing

2.9. Data analysis

3. Results

3.1. Viruses isolated during the outbreak

3.2. Influence of FMD infection on the total lymphocyte counts

3.3. Weight loss

3.4. Haematology

3.5. Phylogenetic relationship of the virus obtained during the outbreak and further elucidation of the relationships of KNP buffalo isolates

3.6. Genetic variation in FMD virus isolates over 167 days

4. Discussion

5. Conclusion

Acknowledgements

References 


\section{Introduction}

Foot-and-mouth disease (FMD) is one of the impediments on the continent of Africa that prevents access to lucrative export markets for animal products. Not only is the disease endemic to large parts of the continent, but serotype distribution and wildlife involvement differ in various parts (Vosloo et al., 2002). In eastern and southern Africa most African buffalo (Syncerus caffer) populations are carriers of the disease and although this status can last up to five years in a single animal, it is possibly not a lifelong infection (Thomson et al., 2003, Thomson and Bastos, 2004 and Vosloo and Thomson, 2004). However, while acutely infected and in the carrier state these animals pose a constant threat to other susceptible cloven hoofed species with which they come into close contact and once the disease is present in domestic animals and no sufficient control measures taken, the disease could be maintained and spread by livestock. The presence of a wildlife maintenance host implies that FMD will not be eradicated from the sub continent unless large numbers of buffalo are eradicated, which is ethically not justifiable. In southern Africa the three South African Territories (SAT) serotypes are prevalent (Vosloo and Thomson, 2004) and the only recent occurrence of serotype $\mathrm{O}$ was caused by an introduction from elsewhere and quickly eradicated (Brückner et al., 2002). The predominant part of South Africa has obtained zoned FMD free status without vaccination from the World Organisation for Animal Health (OIE). The only infected zone is the Kruger National Park (KNP) and adjoining game farms in the north-eastern part of the country where infected buffalo occur. Control of FMD in South Africa is reliant on separation of infected buffalo from susceptible livestock in the rest of the country by wildlife fences, vaccination of domestic animals in a narrow zone along the entire fence of the KNP and other movement control measures. However, experience has shown that outbreaks can still occur once control measures are compromised (Brückner et al., 2002). Buffalo are important for eco-tourism, but FMD carrier animals are not allowed outside the FMD control zone. For this reason several breeding projects are currently underway in southern Africa to breed buffalo calves free of several diseases including FMD (Vosloo and Thomson, 2004) to ensure that genetic diversity is 
maintained and that buffalo can be kept in disease free zones in several southern African countries.

Buffalo calves are born free of infection but become infected with FMD virus by their cohorts as soon as maternal antibody levels decrease. Serological data indicate that they become infected by the three SAT serotypes endemic to sub-Saharan Africa by the time they reach one year of age. This infection is mostly sub-clinical but during the two weeks that acute infection occurs, calves excrete virus in all their excretions and secretions at approximately the same level as cattle (Thomson and Bastos, 2004 and Vosloo and Thomson, 2004). Thereafter virus disappears from all secretions and excretions except for the pharynx where low-level viral replication persists in less or equal than $60 \%$ of individuals and virus can only be isolated from probangs (oropharyngeal scrapings) (Thomson and Bastos, 2004). During persistence, the SAT viruses undergo high rates of mutations, giving rise to new genetic and antigenic variants (Vosloo et al., 1996). It is not clear how virus is transmitted from carrier buffalo to susceptible animals, but for buffalo calves it is believed to spread like a childhood infection once individuals become infected (Thomson et al., 1992). Another possibility for which experimental confirmation is still required, is that virus can be transmitted via sexual transmission since virus was isolated from the semen of a bull of 3.5 years of age which can be accepted to be persistently infected based on its age (Bastos et al., 1999).

Other wildlife species can transmit FMD while in the acute stages of infection and in South Africa at least, the most important seem to be impala (Aepyceros melampus) (Vosloo et al., 2006). Buffalo have been shown unequivocally to infect impala in the KNP (Bastos et al., 2000) and these animals can in turn spread FMD to livestock in close proximity (Vosloo et al., 2006). In other parts of the sub-region, species such as kudu seems to play an important role in disseminating disease (C. Foggin, personal communication, 2003). None of these species become long-term carriers of the disease (Vosloo and Thomson, 2004) although kudu have been shown to carry the disease for up to 140 days post experimental infection (Hedger et al., 1972). Antelope are important since they have the ability to cross fences that normally prevent buffalo from crossing and in Zimbabwe at least one outbreak has been shown to have spread from buffalo to cattle via infected impala (Hargreaves et al., 2004). 
Despite the fact that clinical disease in buffalo is a rare occurrence, this report describes an outbreak of FMD in a group of buffalo that were kept in captivity in the endemic zone of South Africa for an unrelated experiment concerning control of bovine tuberculosis (BTB).

\section{Materials and methods}

\subsection{Study group and description of the outbreak}

Twenty-nine buffalo calves varying in age from ten to twenty months from five different BTB negative herds were captured in the north of the KNP, and transported to enclosures at Skukuza where they were kept for the duration of the BTB vaccine experiment. One group was vaccinated using a Bacille-Calmette-Guérin (BCG) vaccine, while the other served as nonvaccinated controls (see Table 1 for more detail and Fig. 1 for the locations of the herds). The animals were kept together and shared feeding and water troughs. 
Table 1.

Summary of the buffalo included in the BCG vaccine study, their geographic origins and FMD virus isolations post the SAT-1 outbreak

\begin{tabular}{|c|c|c|c|c|c|c|c|c|c|}
\hline & \multirow[t]{2}{*}{ Sex } & \multirow{2}{*}{$\begin{array}{l}\text { Age during FMD } \\
\text { outbreak (months) }\end{array}$} & \multirow[t]{2}{*}{ Herd of origin } & \multirow{2}{*}{$\begin{array}{l}\text { Clinical } \\
\text { FMD }\end{array}$} & \multicolumn{5}{|c|}{ Virus isolated } \\
\hline & & & & & $15 / 05 / 01$ & $19 / 06 / 01$ & 05/09/01 & 09/10/01 & 29/10/01 \\
\hline \multicolumn{10}{|l|}{ Controls } \\
\hline LM 2/z & Male & 30 & Malahlapanga & & & $\mathrm{pb}$ & $\mathrm{pb}$ & $\mathrm{pb}$ & \\
\hline $\mathrm{LM} 4 / \mathrm{z}$ & Female & 24 & Malahlapanga & & & $\mathrm{pb}$ & $\mathrm{pb}$ & & \\
\hline LM 5/z & Female & 20 & Malahlapanga & Yes & & $\mathrm{pb}$ & & & \\
\hline LM 7/z & Female & 24 & Malahlapanga & & & $\mathrm{pb}$ & $\mathrm{pb}$ & $\mathrm{pb}$ & \\
\hline LM 8/z & Female & 24 & Shipande & & & & & & \\
\hline LM 9/z & Female & 20 & Shipande & Yes & & $\mathrm{pb}$ & $\mathrm{pb}$ & & \\
\hline LM 11/z & Female & 30 & Shipande & Yes & Epith & $\mathrm{pb}, \mathrm{ns}$ & fs & $\mathrm{pb}$ & $\mathrm{pb}$ \\
\hline LM 12/z & Female & 24 & Shipande & & & $\mathrm{pb}$ & $\mathrm{pb}, \mathrm{fs}$ & & \\
\hline LM 15/z & Female & 30 & Shipande & Yes & & $\mathrm{pb}$ & $\mathrm{pb}$ & & $\mathrm{pb}$ \\
\hline LM 20/z & Female & 30 & Klopperfontein & & & $\mathrm{pb}$ & & & \\
\hline LM 24/z & Male & 24 & Klopperfontein & & & $\mathrm{pb}$ & $\mathrm{pb}$ & & \\
\hline LM 28/z & Male & 30 & Tsombeyeni & Yes & & & $\mathrm{pb}, \mathrm{fs}$ & fs & \\
\hline
\end{tabular}




\begin{tabular}{|c|c|c|c|c|c|c|c|c|c|}
\hline & Sex & Age during FMD & Herd of origin & $\begin{array}{l}\text { Clinical } \\
\text { FMD }\end{array}$ & \multicolumn{5}{|c|}{ Virus isolated } \\
\hline & & & & & $15 / 05 / 01$ & 19/06/01 & 05/09/01 & 09/10/01 & 29/10/01 \\
\hline LM 31/z & Female & 20 & Tsombeyeni & & & & $\mathrm{pb}, \mathrm{fs}$ & $\mathrm{pb}$ & \\
\hline LM 33/z & Male & 20 & Gadzingwe & & & & $\mathrm{pb}$ & & \\
\hline \multicolumn{10}{|l|}{ Vaccinates } \\
\hline LM 1/z & Male & 30 & Malahlapanga & & & $\mathrm{pb}$ & $\mathrm{pb}$ & $\mathrm{pb}$ & \\
\hline LM 3/z & Female & 30 & Malahlapanga & & & & & $\mathrm{pb}$ & \\
\hline LM 10/z & Male & 24 & Shipande & & & $\mathrm{pb}$ & $\mathrm{pb}, \mathrm{fs}$ & fs & \\
\hline LM 14/z & Female & 30 & Shipande & & & & & & \\
\hline LM 16/z & Female & 20 & Klopperfontein & & & & fs & & fs \\
\hline LM 18/z & Male & 20 & Klopperfontein & & & & $\mathrm{pb}, \mathrm{fs}$ & $\mathrm{pb}$ & \\
\hline LM 19/z & Female & 30 & Klopperfontein & Yes & & $\mathrm{pb}$ & $\mathrm{pb}$ & $\mathrm{pb}$ & \\
\hline LM 21/z & Male & 30 & Klopperfontein & & & & $\mathrm{pb}$ & & \\
\hline LM 23/z & Female & 24 & Klopperfontein & & & $\mathrm{pb}$ & & & \\
\hline LM 25/z & Female & 24 & Tsombeyeni & & & $\mathrm{pb}$ & $\mathrm{pb}$ & & \\
\hline LM 27/z & Female & 30 & Tsombeyeni & & & & $\mathrm{pb}, \mathrm{fs}$ & & fs \\
\hline
\end{tabular}




\begin{tabular}{|c|c|c|c|c|c|c|c|c|c|}
\hline & Sex & $\begin{array}{l}\text { Age during FMD } \\
\text { outbreak (months) }\end{array}$ & Herd of origin & $\begin{array}{l}\text { Clinical } \\
\text { FMD }\end{array}$ & \multicolumn{5}{|c|}{ Virus isolated } \\
\hline & & & & & 15/05/01 & 19/06/01 & 05/09/01 & 09/10/01 & 29/10/01 \\
\hline LM 29/z & Female & 24 & Tsombeyeni & & & $\mathrm{pb}$ & & $\mathrm{pb}$ & \\
\hline LM 30/z & Male & 24 & Tsombeyeni & & & $\mathrm{pb}$ & & & \\
\hline LM 32/z & Female & 24 & Gadzingwe & & & $\mathrm{pb}$ & $\mathrm{pb}$ & & \\
\hline LM 35/z & Female & 20 & Gadzingwe & & & & & & \\
\hline
\end{tabular}

Epith: epithelium; pb: probing; fs: pharyngeal swab; ns: nasal swab. 


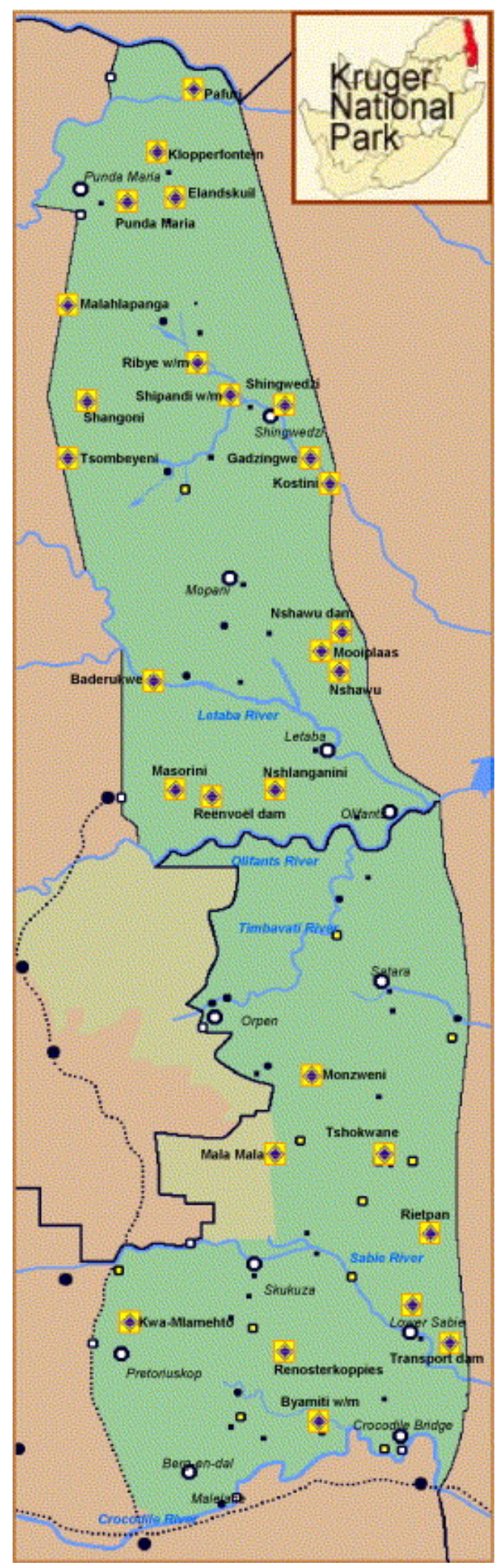

Fig. 1. Map of the Kruger Park. Locations of the herds (provided in Table 1 and Table 3) are indicated on the map. 
Calves were monitored daily to ensure their general health and welfare and faecal samples were collected weekly to determine internal parasite infestation. The buffalo

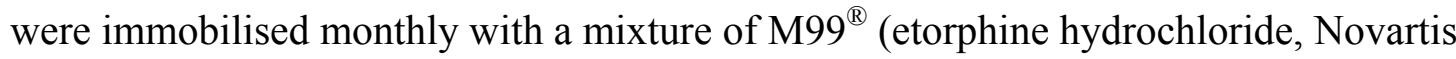
SA Ltd, Animal Health) and Stresnil ${ }^{\circledR}$ (Azaperone, Janssen Animal Health) at standard capture doses, weighed and blood samples were collected via jugular venipuncture and serum as well as whole blood collected in EDTA tubes for serological evaluation of FMD infection and full blood counts respectively to monitor health status. Animals were regularly tested for internal parasites and received treatment accordingly. Amprolium 2\% was used to treat the water when the coccidial counts increased to more than $5000 \mathrm{~g}^{-1}$ of pooled faecal material. Dectomax $1 \%$ injectable was used to rid buffaloes of helminth infestations.

The index case in the FMD outbreak occurred six months after the buffalo calves arrived at the bomas. A 30-month-old heifer (LM11/z) showed obvious discomfort of the oral cavity together with constant chewing on 6 May 2001. White foam appeared at the corners of the mouth but drooling of saliva was not seen. She was anorexic, did not show interest in her food and soaked her mouth in the water trough. When this animal was immobilised two days later (8 May 2001), she had severe lesions on the dorsal surface of the tongue and hard palate. The epithelium of the dorsal and lateral aspects of the tongue surface had sloughed, and some of the epithelial tissues were collected for FMD virus isolation. On closer examination, five more animals (LM5/z, LM9/z, LM15/z, LM19/z and LM28/z) were found to have lesions in the mouth. These occurred on the tongue, insides of the cheeks and, in one case, on the hard palate. Some lesions were large $(70 \mathrm{~mm} \times 30 \mathrm{~mm}$ ), foul smelling and the affected epithelium was brittle and came off in granules. The majority of the calves also showed depression, pyrexia and moderate anorexia. Within a week, ulcers/erosions formed with rounded epithelial edges and a clear pink floor. After two weeks the tongue lesions were visible as pale, weakly circumscribed areas with poorly developed papillae. Foot lesions did not occur in any of the animals. The animals were all slaughtered between 29 October to 2 November 2001 as originally planned for the BTB vaccination experiment.

\subsection{Viruses included in the study}


A number of viruses isolated during and after the outbreak in the experimental animals were used for phylogenetic analysis and the 1D gene sequenced to determine the amount of genetic changes in this gene during persistence (Table 2). Additional buffalo isolates obtained from the KNP were included to determine the phylogenetic relationship with the outbreak isolate and to further elucidate the epidemiology of FMD in buffalo populations in the KNP (Table 3). Reference strains from published work were included as well as 23 new isolates sequenced for this study.

Table 2.

FMD virus isolates included in the analysis of genetic variation obtained from buffalo during and after the outbreak

\begin{tabular}{|c|c|c|c|c|c|}
\hline $\begin{array}{l}\text { Buffalo } \\
\text { No. }\end{array}$ & $\begin{array}{l}\text { Sampling } \\
\text { date }\end{array}$ & $\begin{array}{l}\text { Days post first } \\
\text { clinical case }\end{array}$ & $\begin{array}{l}\text { Virus } \\
\text { name }\end{array}$ & Specimen & $\begin{array}{l}\text { Passage } \\
\text { history }\end{array}$ \\
\hline \multirow[t]{5}{*}{ LM 11/z } & 08/05/2001 & 2 & $\mathrm{KNP} / 01 / 01$ & Epithelium & PK1 \\
\hline & 07/06/2001 & 32 & $\mathrm{KNP} / 34 / 01$ & Probang & PK1 RS1 \\
\hline & 05/09/2001 & 122 & $\mathrm{KNP} / 23 / 01$ & $\begin{array}{l}\text { Pharyngeal } \\
\text { swap }\end{array}$ & PK1 \\
\hline & $12 / 10 / 2001$ & 159 & $\mathrm{KNP} / 38 / 01$ & Probang & PK1 RS1 \\
\hline & $29 / 10 / 2001$ & 176 & $\mathrm{KNP} / 33 / 01$ & Probang & PK1 RS1 \\
\hline \multirow[t]{3}{*}{ LM 15/z } & $07 / 06 / 2001$ & 32 & $\mathrm{KNP} / 5 / 01$ & Probang & PK1 RS1 \\
\hline & 05/09/2001 & 122 & $\mathrm{KNP} / 24 / 01$ & Probang & PK1 \\
\hline & $29 / 10 / 2001$ & 176 & $\mathrm{KNP} / 35 / 01$ & Probang & PK1 RS1 \\
\hline \multirow[t]{3}{*}{ LM 7/z } & $07 / 06 / 2001$ & 32 & $\mathrm{KNP} / 14 / 01$ & Probang & PK1 \\
\hline & 05/09/2001 & 122 & $\mathrm{KNP} / 20 / 01$ & Probang & PK1 \\
\hline & $12 / 10 / 2001$ & 159 & $\mathrm{KNP} / 36 / 01$ & Probang & PK1 RS1 \\
\hline
\end{tabular}

PK1: passaged once on primary pig kidney cells. RS1: passaged once on IB-RS2 (Instituto Biologico Rim Suino) cells. 
Table 3.

Summary of the SAT-1 buffalo isolates included in the phylogenetic analysis from the Kruger National Park and surrounding countries

\begin{tabular}{|c|c|c|c|c|c|c|}
\hline Isolate & Country & Location & $\begin{array}{l}\text { Species of } \\
\text { origin }\end{array}$ & Grid ref & $\begin{array}{l}\text { Sampling } \\
\text { year }\end{array}$ & $\begin{array}{l}\text { Gen Bank } \\
\text { accession No. }\end{array}$ \\
\hline \multicolumn{7}{|c|}{ Northern Kruger National Park } \\
\hline $\begin{array}{l}\mathrm{SAR} / 9 / 81 \text { (Bastos et al., } \\
\text { 2001) }\end{array}$ & $\begin{array}{l}\text { South } \\
\text { Africa }\end{array}$ & Pafuri & Impala & $\begin{array}{l}30^{\circ} 00^{\prime} \mathrm{E} \\
24^{\circ} 50^{\prime} \mathrm{S}\end{array}$ & 1981 & AF056511 \\
\hline $\begin{array}{l}\text { KNP/10/86 (Bastos et al., } \\
\text { 2001) }\end{array}$ & $\begin{array}{l}\text { South } \\
\text { Africa }\end{array}$ & Punda Maria & Buffalo & $\begin{array}{l}31^{\circ} 10^{\prime} \mathrm{E} \\
22^{\circ} 42^{\prime} \mathrm{S}\end{array}$ & 1986 & AY396735 \\
\hline $\mathrm{KNP} / 1 / 87^{\mathbf{a}}$ & $\begin{array}{l}\text { South } \\
\text { Africa }\end{array}$ & Mooiplaas & Buffalo & $\begin{array}{l}31^{\circ} 27^{\prime} \mathrm{E} \\
23^{\circ} 34^{\prime} \mathrm{S}\end{array}$ & 1987 & DQ780594 \\
\hline $\mathrm{KNP} / 2 / 87^{\mathrm{a}}$ & $\begin{array}{l}\text { South } \\
\text { Africa }\end{array}$ & Mooiplaas & Buffalo & $\begin{array}{l}31^{\circ} 27^{\prime} \mathrm{E} \\
23^{\circ} 34^{\prime} \mathrm{S}\end{array}$ & 1987 & DQ780595 \\
\hline $\mathrm{KNP} / 1 / 90^{\mathrm{a}}$ & $\begin{array}{l}\text { South } \\
\text { Africa }\end{array}$ & Reënvoëldam & Buffalo & $\begin{array}{l}31^{\circ} 20^{\prime} \mathrm{E} \\
24^{\circ} 00^{\prime} \mathrm{S}\end{array}$ & 1990 & DQ780596 \\
\hline $\mathrm{KNP} / 24 / 91^{\mathrm{a}}$ & $\begin{array}{l}\text { South } \\
\text { Africa }\end{array}$ & Nshawu & Buffalo & $\begin{array}{l}31^{\circ} 29^{\prime} \mathrm{E} \\
23^{\circ} 35^{\prime} \mathrm{S}\end{array}$ & 1991 & DQ780577 \\
\hline $\mathrm{KNP} / 174 / 91^{\mathrm{a}}$ & $\begin{array}{l}\text { South } \\
\text { Africa }\end{array}$ & Nshlanganini & Buffalo & $\begin{array}{l}31^{\circ} 26^{\prime} \mathrm{E} \\
23^{\circ} 55^{\prime} \mathrm{S}\end{array}$ & 1991 & DQ780597 \\
\hline $\mathrm{KNP} / 29 / 92^{\mathrm{a}}$ & $\begin{array}{l}\text { South } \\
\text { Africa }\end{array}$ & Pafuri & Buffalo & $\begin{array}{l}31^{\circ} 13^{\prime} \mathrm{E} \\
22^{\circ} 25^{\prime} \mathrm{S}\end{array}$ & 1992 & DQ780583 \\
\hline $\mathrm{KNP} / 35 / 92^{\mathrm{a}}$ & South & Shingwedzi & Buffalo & $31^{\circ} 13^{\prime} \mathrm{E}$ & 1992 & DQ780584 \\
\hline
\end{tabular}




\begin{tabular}{|c|c|c|c|c|c|c|}
\hline Isolate & Country & Location & $\begin{array}{l}\text { Species of } \\
\text { origin }\end{array}$ & Grid ref & $\begin{array}{l}\text { Sampling } \\
\text { year }\end{array}$ & $\begin{array}{l}\text { Gen Bank } \\
\text { accession No. }\end{array}$ \\
\hline & Africa & & & $22^{\circ} 25^{\prime} \mathrm{S}$ & & \\
\hline $\mathrm{KNP} / 36 / 92^{\mathrm{a}}$ & $\begin{array}{l}\text { South } \\
\text { Africa }\end{array}$ & Shingwedzi & Buffalo & $\begin{array}{l}31^{\circ} 24^{\prime} \mathrm{E} \\
23^{\circ} 00^{\prime} \mathrm{S}\end{array}$ & 1992 & DQ780585 \\
\hline $\mathrm{KNP} / 45 / 92^{\mathrm{a}}$ & $\begin{array}{l}\text { South } \\
\text { Africa }\end{array}$ & Shingwedzi & Buffalo & $\begin{array}{l}31^{\circ} 32^{\prime} \mathrm{E} \\
23^{\circ} 20^{\prime} \mathrm{S}\end{array}$ & 1992 & DQ780582 \\
\hline $\begin{array}{l}\text { KNP/17/96 (Bastos et al., } \\
\text { 2001) }\end{array}$ & $\begin{array}{l}\text { South } \\
\text { Africa }\end{array}$ & Kostini & Buffalo & $\begin{array}{l}31^{\circ} 32^{\prime} \mathrm{E} \\
23^{\circ} 18^{\prime} \mathrm{S}\end{array}$ & 1996 & AF301431 \\
\hline $\begin{array}{l}\mathrm{KNP} / 75 / 98 \text { (Bastos et al., } \\
\text { 2001) }\end{array}$ & $\begin{array}{l}\text { South } \\
\text { Africa }\end{array}$ & Ribye Waterhole & Buffalo & $\begin{array}{l}31^{\circ} 16^{\prime} \mathrm{E} \\
22^{\circ} 59^{\prime} \mathrm{S}\end{array}$ & 1998 & AF137406 \\
\hline $\mathrm{KNP} / 886 / 98^{\mathrm{a}}$ & $\begin{array}{l}\text { South } \\
\text { Africa }\end{array}$ & $\begin{array}{l}\text { Shipandi } \\
\text { Windmill }\end{array}$ & Buffalo & $\begin{array}{l}31^{\circ} 20^{\prime} \mathrm{E} \\
23^{\circ} 06^{\prime} \mathrm{S}\end{array}$ & 1998 & DQ780578 \\
\hline $\mathrm{KNP} / 893 / 98^{\mathrm{a}}$ & $\begin{array}{l}\text { South } \\
\text { Africa }\end{array}$ & $\begin{array}{l}\text { Shipandi } \\
\text { Windwill }\end{array}$ & Buffalo & $\begin{array}{l}31^{\circ} 20^{\prime} \mathrm{E} \\
23^{\circ} 06^{\prime} \mathrm{S}\end{array}$ & 1998 & DQ780579 \\
\hline $\mathrm{KNP} / 1584 / 98^{\mathbf{a}}$ & $\begin{array}{l}\text { South } \\
\text { Africa }\end{array}$ & Baderukwe spruit & Buffalo & $\begin{array}{l}31^{\circ} 10^{\prime} \mathrm{E} \\
23^{\circ} 43^{\prime} \mathrm{S}\end{array}$ & 1998 & DQ780593 \\
\hline $\mathrm{KNP} / 01 / 01^{\mathrm{a}}$ & $\begin{array}{l}\text { South } \\
\text { Africa }\end{array}$ & $\begin{array}{l}\text { Shipandi } \\
\text { Windmill }\end{array}$ & Buffalo & $\begin{array}{l}31^{\circ} 19^{\prime} \mathrm{E} \\
23^{\circ} 06^{\prime} \mathrm{S}\end{array}$ & 2001 & DQ780586 \\
\hline $\mathrm{KNP} / 2 / 02^{\mathbf{a}}$ & $\begin{array}{l}\text { South } \\
\text { Africa }\end{array}$ & Elandskuil & Buffalo & $\begin{array}{l}31^{\circ} 09^{\prime} \mathrm{E} \\
22^{\circ} 42^{\prime} \mathrm{S}\end{array}$ & 2002 & DQ780587 \\
\hline $\mathrm{KNP} / 3 / 02^{\mathrm{a}}$ & $\begin{array}{l}\text { South } \\
\text { Africa }\end{array}$ & Elandskuil & Buffalo & $\begin{array}{l}31^{\circ} 09^{\prime} \mathrm{E} \\
22^{\circ} 42^{\prime} \mathrm{S}\end{array}$ & 2002 & DQ780588 \\
\hline
\end{tabular}




\begin{tabular}{|c|c|c|c|c|c|c|}
\hline Isolate & Country & Location & $\begin{array}{l}\text { Species of } \\
\text { origin }\end{array}$ & Grid ref & $\begin{array}{l}\text { Sampling } \\
\text { year }\end{array}$ & $\begin{array}{l}\text { Gen Bank } \\
\text { accession No. }\end{array}$ \\
\hline $\mathrm{KNP} / 3 / 03^{\mathrm{a}}$ & $\begin{array}{l}\text { South } \\
\text { Africa }\end{array}$ & Pafuri & Buffalo & $\begin{array}{l}31^{\circ} 18^{\prime} \mathrm{E} \\
22^{\circ} 62^{\prime} \mathrm{S}\end{array}$ & 2003 & DQ780589 \\
\hline $\mathrm{KNP} / 7 / 03^{\mathrm{a}}$ & $\begin{array}{l}\text { South } \\
\text { Africa }\end{array}$ & Shangoni & Buffalo & $\begin{array}{l}31^{\circ} 05^{\prime} \mathrm{E} \\
22^{\circ} 39^{\prime} \mathrm{S}\end{array}$ & 2003 & DQ780590 \\
\hline $\mathrm{KNP} / 10 / 03^{\mathrm{a}}$ & $\begin{array}{l}\text { South } \\
\text { Africa }\end{array}$ & Nshawu dam & Buffalo & $\begin{array}{l}31^{\circ} 29^{\prime} \mathrm{E} \\
23^{\circ} 33^{\prime} \mathrm{S}\end{array}$ & 2003 & DQ780591 \\
\hline $\mathrm{KNP} / 11 / 03^{\mathrm{a}}$ & $\begin{array}{l}\text { South } \\
\text { Africa }\end{array}$ & Masorini & Buffalo & $\begin{array}{l}31^{\circ} 15^{\prime} \mathrm{E} \\
23^{\circ} 26^{\prime} \mathrm{S}\end{array}$ & 2003 & DQ780592 \\
\hline \multicolumn{7}{|c|}{ Southern Kruger National Park } \\
\hline $\begin{array}{l}\text { KNP/6/86 (Bastos et al., } \\
\text { 2001) }\end{array}$ & $\begin{array}{l}\text { South } \\
\text { Africa }\end{array}$ & Tshokwane & Buffalo & $\begin{array}{l}31^{\circ} 51^{\prime} \mathrm{E} \\
24^{\circ} 47^{\prime} \mathrm{S}\end{array}$ & 1986 & AY274484 \\
\hline $\begin{array}{l}\mathrm{KNP} / 20 / 89 \text { (Bastos et al., } \\
\text { 2001) }\end{array}$ & $\begin{array}{l}\text { South } \\
\text { Africa }\end{array}$ & Kwa Mlamehto & Buffalo & $\begin{array}{l}31^{\circ} 12^{\prime} \mathrm{E} \\
25^{\circ} 06^{\prime} \mathrm{S}\end{array}$ & 1989 & AY274489 \\
\hline $\begin{array}{l}\mathrm{KNP} / 148 / 91 \text { (Bastos et al., } \\
\text { 2001) }\end{array}$ & $\begin{array}{l}\text { South } \\
\text { Africa }\end{array}$ & Renosterkoppies & Buffalo & $\begin{array}{l}31^{\circ} 36^{\prime} \mathrm{E} \\
25^{\circ} 07^{\prime} \mathrm{S}\end{array}$ & 1991 & AF023513 \\
\hline $\begin{array}{l}\text { KNP/196/91 (Bastos et al., } \\
\text { 2001) }\end{array}$ & $\begin{array}{l}\text { South } \\
\text { Africa }\end{array}$ & $\begin{array}{l}\text { Biyamiti } \\
\text { Windmill }\end{array}$ & Buffalo & $\begin{array}{l}31^{\circ} 38^{\prime} \mathrm{E} \\
25^{\circ} 17^{\prime} \mathrm{S}\end{array}$ & 1991 & AF056506 \\
\hline $\begin{array}{l}\mathrm{KNP} / 8 / 95 \text { (Bastos et al., } \\
\text { 2001) }\end{array}$ & $\begin{array}{l}\text { South } \\
\text { Africa }\end{array}$ & Monzweni & Buffalo & $\begin{array}{l}31^{\circ} 38^{\prime} \mathrm{E} \\
24^{\circ} 34^{\prime} \mathrm{S}\end{array}$ & 1995 & AF056503 \\
\hline KNP/14/95 (Bastos et al., & South & Monzweni & Buffalo & $31^{\circ} 38^{\prime} \mathrm{E}$ & 1995 & AF056504 \\
\hline
\end{tabular}




\begin{tabular}{|c|c|c|c|c|c|c|}
\hline Isolate & Country & Location & $\begin{array}{l}\text { Species of } \\
\text { origin }\end{array}$ & Grid ref & $\begin{array}{l}\text { Sampling } \\
\text { year }\end{array}$ & $\begin{array}{l}\text { Gen Bank } \\
\text { accession No. }\end{array}$ \\
\hline 2001) & Africa & & & $24^{\circ} 34^{\prime} \mathrm{S}$ & & \\
\hline $\begin{array}{l}\text { KNP/17/95 (Bastos et al., } \\
\text { 2001) }\end{array}$ & $\begin{array}{l}\text { South } \\
\text { Africa }\end{array}$ & Monzweni & Buffalo & $\begin{array}{l}31^{\circ} 38^{\prime} \mathrm{E} \\
24^{\circ} 34^{\prime} \mathrm{S}\end{array}$ & 1995 & AF056505 \\
\hline $\begin{array}{l}\text { KNP/41/95 (Bastos et al., } \\
\text { 2001) }\end{array}$ & $\begin{array}{l}\text { South } \\
\text { Africa }\end{array}$ & Monzweni & Buffalo & $\begin{array}{l}31^{\circ} 38^{\prime} \mathrm{E} \\
24^{\circ} 34^{\prime} \mathrm{S}\end{array}$ & 1995 & AF056508 \\
\hline $\begin{array}{l}\text { KNP/2/96 (Bastos et al., } \\
\text { 2001) }\end{array}$ & $\begin{array}{l}\text { South } \\
\text { Africa }\end{array}$ & Mala Mala & Buffalo & $\begin{array}{l}31^{\circ} 33^{\prime} \mathrm{E} \\
24^{\circ} 55^{\prime} \mathrm{S}\end{array}$ & 1996 & AF056502 \\
\hline $\begin{array}{l}\mathrm{KNP} / 22 / 96 \text { (Bastos et al., } \\
\text { 2001) }\end{array}$ & $\begin{array}{l}\text { South } \\
\text { Africa }\end{array}$ & Lower Sabie & Buffalo & $\begin{array}{l}31^{\circ} 55^{\prime} \mathrm{E} \\
25^{\circ} 07^{\prime} \mathrm{S}\end{array}$ & 1996 & AF056507 \\
\hline $\mathrm{KNP} / 397 / 98^{\mathrm{a}}$ & $\begin{array}{l}\text { South } \\
\text { Africa }\end{array}$ & Rietpan & Buffalo & $\begin{array}{l}31^{\circ} 57^{\prime} \mathrm{E} \\
04^{\circ} 54^{\prime} \mathrm{S}\end{array}$ & 1998 & DQ780580 \\
\hline $\mathrm{KNP} / 400 / 98^{\mathrm{a}}$ & $\begin{array}{l}\text { South } \\
\text { Africa }\end{array}$ & Rietpan & Buffalo & $\begin{array}{l}31^{\circ} 57^{\prime} \mathrm{E} \\
24^{\circ} 54^{\prime} \mathrm{S}\end{array}$ & 1998 & DQ780581 \\
\hline $\mathrm{KNP} / 1 / 05^{\mathrm{a}}$ & $\begin{array}{l}\text { South } \\
\text { Africa }\end{array}$ & Biyamiti spruit & Buffalo & $\begin{array}{l}31^{\circ} 05^{\prime} \mathrm{E} \\
25^{\circ} 15^{\prime} \mathrm{S}\end{array}$ & 2005 & DQ780599 \\
\hline $\mathrm{KNP} / 5 / 05^{\mathrm{a}}$ & $\begin{array}{l}\text { South } \\
\text { Africa }\end{array}$ & Transportdam & Buffalo & $\begin{array}{l}31^{\circ} 52^{\prime} \mathrm{E} \\
25^{\circ} 13^{\prime} \mathrm{S}\end{array}$ & 2005 & DQ780598 \\
\hline \multicolumn{7}{|l|}{ Other countries } \\
\hline $\begin{array}{l}\text { BOT } / 8 / 98 \text { (Bastos et al., } \\
\text { 2001) }\end{array}$ & Botswana & Nxaraga & Buffalo & $\begin{array}{l}23^{\circ} 15^{\prime} \mathrm{E} \\
19^{\circ} 40^{\prime} \mathrm{S}\end{array}$ & 1998 & AF017403 \\
\hline
\end{tabular}




\begin{tabular}{|l|l|l|l|l|l|l|}
\hline Isolate & Country & Location & $\begin{array}{l}\text { Species of } \\
\text { origin }\end{array}$ & Grid ref & $\begin{array}{l}\text { Sampling } \\
\text { year }\end{array}$ & $\begin{array}{l}\text { Gen Bank } \\
\text { accession No. }\end{array}$ \\
\hline $\begin{array}{l}\text { MAL/1/85 (Bastos et al., } \\
\mathbf{2 0 0 1}\end{array}$ & Malawi & Kasungu NP & Buffalo & $\begin{array}{l}33^{\circ} 30^{\prime} \mathrm{E} \\
13^{\circ} 00^{\prime} \mathrm{S}\end{array}$ & 1985 & AF056509 \\
\hline $\begin{array}{l}\text { NAM/288/98 (Bastos et } \\
\text { al., 2001) }\end{array}$ & Namibia & West Caprivi GR & Buffalo & $\begin{array}{l}21^{\circ} 50^{\prime} \mathrm{E} \\
18^{\circ} 15^{\prime} \mathrm{S}\end{array}$ & 1998 & AF137409 \\
\hline $\begin{array}{l}\text { NAM/307/98 (Bastos et } \\
\text { al., 2001) }\end{array}$ & Namibia & West Caprivi GR & Buffalo & $\begin{array}{l}21^{\circ} 50^{\prime} \mathrm{E} \\
08^{\circ} 15^{\prime} \mathrm{S}\end{array}$ & 1998 & AF301433 \\
\hline $\begin{array}{l}\text { ZAM/2/93 (Bastos et al., } \\
\mathbf{2 0 0 1})\end{array}$ & Zambia & Kafue NP & Buffalo & $\begin{array}{l}26^{\circ} 00^{\prime} \mathrm{E} \\
6^{\circ} 30^{\prime} \mathrm{S}\end{array}$ & 1993 & AF056514 \\
\hline $\begin{array}{l}\text { ZIM/2/90 (Bastos et al., } \\
\mathbf{2 0 0 1})\end{array}$ & Zimbabwe & $\begin{array}{l}\text { Chirisa Safari } \\
\text { area }\end{array}$ & Buffalo? & $\begin{array}{l}28^{\circ} 15^{\prime} \mathrm{E} \\
18^{\circ} 00^{\prime} \mathrm{S}\end{array}$ & 1990 & AF056518 \\
\hline $\begin{array}{l}\text { ZIM/3/88 (Bastos et al., } \\
\mathbf{2 0 0 1})\end{array}$ & Zimbabwe & Hwange NP & Buffalo & $\begin{array}{l}27^{\circ} 00^{\prime} \mathrm{E} \\
19^{\circ} 00^{\prime} \mathrm{S}\end{array}$ & 1988 & AF056520 \\
\hline
\end{tabular}

GR: Game Reserve; NP: National Park.

${ }^{a}$ This study. 


\subsection{Haematology}

Whole blood was collected from the jugular vein in EDTA tubes and submitted for full blood counts within six hours of collection. A coulter counter (Coulter T-890, Beckman) was used to process the whole blood samples and results were recorded, printed, and stored for each individual animal on a monthly basis.

\subsection{Collection of clinical material}

Epithelium was removed from lesions and transported in phosphate buffered saline (PBS). A probang cup (Sutmoller and Gaggero, 1965) and/or pharyngeal brush were inserted into the oesophageo-pharyngeal region of each immobilized buffalo to collect epithelium and emptied and rinsed into $10 \mathrm{~mL}$ PBS. These samples were frozen and stored in liquid nitrogen directly after removal from the animals and transported to the Exotic Diseases Division, Onderstepoort Veterinary Institute.

\subsection{Virus isolation on cell cultures}

The FMD isolates were obtained by preparing $10 \%$ suspensions (w/v) of buffalo probangs and epithelium specimens according to standard procedures. Primary pig kidney (PK) cells were inoculated with the suspensions, incubated at $37{ }^{\circ} \mathrm{C}$ for $60 \mathrm{~min}$, the medium decanted and fresh medium added where after cell culture fluids were harvested as soon as cytopathogenic effect (CPE) was observed but not for less than $24 \mathrm{~h}$. If CPE was not observed within this period, the whole cell harvest was passaged blind once and further observed for $24 \mathrm{~h}$. Clinical material was scored as negative for FMD virus if no CPE was observed after this 48-h incubation. Positive samples were further propagated on IB-RS2 (Instituto Biologico Rim Suino) cells and typed.

\subsection{RNA extraction and cDNA synthesis}

RNA was extracted from cell culture material (see Table 2 for passage history of isolates included in the study and Table 3 for the additional isolates used in the phylogenetic analysis) using a silica/guanidinium-based nucleic acid extraction method of Boom et al. (1990). The viral RNA template was reverse transcribed at $42{ }^{\circ} \mathrm{C}$ for $1 \mathrm{~h}$ using $10 \mathrm{U}$ of 
AMV reverse transcriptase (Promega) and the P1 primer of Beck and Strohmaier (1987) as described previously by Bastos (1998).

\subsection{Polymerase chain reaction}

Genomic amplification of the 1D gene of FMDV was performed using modified primers P1 [5'-GAA GGG CCC AGG GTT GGA CTC-3'] and VP3AB [5'-CAC TGC TAC CAC TCR GAG TG-3']. PCR reactions were performed in $50 \mu \mathrm{L}$ volumes in the presence of $3 \mu \mathrm{L}$ cDNA, $200 \mu \mathrm{M}$ dNTPs, $2.5 \mathrm{U}$ Taq polymerase (Roche), $1 \times$ Taq buffer, $1.5 \mathrm{mM}$ $\mathrm{MgCl}_{2}$ and $25 \mathrm{pmol}$ of each primer. The template was amplified following 40 cycles of denaturation at $96{ }^{\circ} \mathrm{C}$ for $12 \mathrm{~s}$, annealing at $56{ }^{\circ} \mathrm{C}$ for $30 \mathrm{~s}$ and extension at $72{ }^{\circ} \mathrm{C}$ for $30 \mathrm{~s}$. Amplification products were separated on a 1.5\% agarose gel and visualised by ethidium bromide staining and UV irradiation.

\subsection{PCR product purification and nucleotide sequencing}

PCR products of the correct size were excised from a 1.5\% agarose gel and purified using the Qia Quick Gel Extraction Kit (Qiagen), and eluted in $20 \mu \mathrm{L}$ of elution buffer. Purified PCR products were sequenced using the Big Dye ${ }^{\circledR}$ version 3.0 Cycle Sequencing Kit and the ABI Prism 310 Genetic Analyser (Applied Biosystems), and the same primers as for amplification. The full 1D sequence as well as 46 nucleotides of the C-terminal part of VP3 were generated. For the phylogenetic analysis, 318 nucleotides of the C-terminal part of $1 \mathrm{D}$ and sequences from $2 \mathrm{~A}$ were used.

\subsection{Data analysis}

Nucleotide sequences generated were aligned with additional sequences indicated in Table 3 using DAPSA (Harley, 2001). Maximum likelihood analysis of the aligned sequences were carried out in PAUP* (Swofford, 2002) assuming an HKY model of nucleotide substitution and rate heterogeneity. Confidence levels were assessed using a Neighbor-joining algorithm with 1000 bootstrap replications and a 50\% consensus tree constructed. High-resolution diagrams reconstructing the genealogies of the sequences isolated from the buffalo were constructed using the TCS software (Clement et al., 2000) assuming missing data was a fifth state. The rate of nucleotide substitution per site per day over the course of the outbreak in the buffalo was estimated using the software 
BEAST (Drummond et al., 2002). We assumed a constant viral population size consistent with a long-term infection, a strict clock, and an HKY model of nucleotide substitution with no rate heterogeneity (since the level of divergence within the buffalo sequences is extremely low).

\section{Results}

\subsection{Viruses isolated during the outbreak}

Serology results indicated that by October 2000 when the BTB experiment started, all the buffalo calves had high titres of mostly more than $2.4 \log _{10}$ against all three SAT serotypes (results not shown). No clinical disease was observed during this time. During May 2001, five of the fourteen animals in the control group that was not vaccinated with BCG were clinically affected, compared to one of the fifteen animals that were vaccinated. The prevalence of FMD in the vaccinated and control groups is not significantly different (Fishers exact test: $p=0.187$ ). SAT-1 virus was isolated only from one epithelium sample on Day 2, and intermittently from probangs and pharyngeal swabs of 27/29 animals over the duration of the experiment (Table 1). The index case (LM11/z) was the only animal from which virus could be isolated for the duration of the experiment. One animal from each experimental group (LM8/z control and LM35/z vaccinated animal) was negative for virus isolation over the duration of the outbreak (Table 1). The percentage positive animals decreased over time with $69.2 \%$ of probangs and pharyngeal swabs positive on Day 32, 69\% on Day 122, 37.9\% on Day 159, and $17.4 \%$ on Day 176. From all 153 samples where both probang and pharyngeal swabs were taken for virus isolation, in 7 (4.6\%) instances could virus be isolated from both, 44 (28.8\%) probangs only yielded virus, compared to $8(5.2 \%)$ from the pharyngeal swabs. Clearly the pharyngeal swabs are not the choice for virus isolation during and after outbreaks, however, it added to the list of virus isolations in a few cases.

\subsection{Influence of FMD infection on the total lymphocyte counts}

The total lymphocyte counts from both the BCG vaccinated and control groups were not significantly different over the period of the FMD SAT-1 outbreak (Fig. 2). At Day 0, the average counts were approximately $7.5 \times 10^{6} \mathrm{~mL}^{-1}$ (Fig. 2) showing a significant 
lymphopenia at Day 35 of about $5 \times 10^{6} \mathrm{~mL}^{-1}$. The lowest counts were observed at Day 65 after which the number of lymphocytes started increasing at Day 101. At Days 126 and 192 the total lymphocyte counts have increased to $7 \times 10^{6} \mathrm{~mL}^{-1}$ not significantly different to their values on Day $0(t=0.9, p=0.38$, Fig. 2$)$.

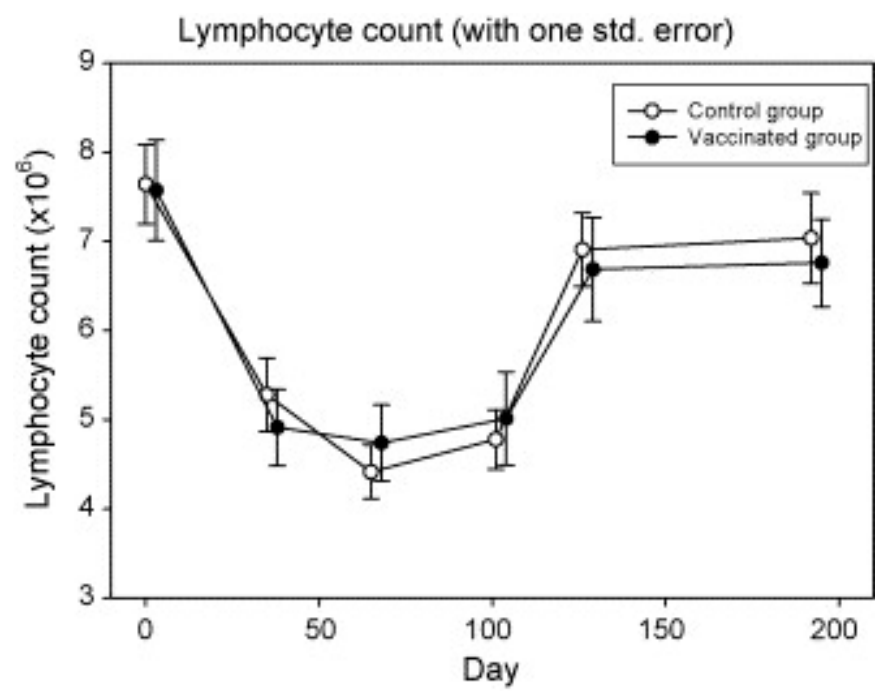

Fig. 2. Lymphocyte counts over the 192-day period of the study. Vaccinated (filled circles) and control groups (unfilled circles) are offset by a small time interval to avoid superimposition.

\subsection{Weight loss}

The maximum weight loss for each animal was calculated, and the relationship between initial weight and maximum weight loss expressed both in absolute weight loss, and as a proportion of the initial weight examined. Both are significantly related to initial weight (GLM, $F(1,27)=34.41, p<0.0001$; and $F(1,27)=17.08, p<0.0001)$. Average maximum weight loss across all infected individuals was $13.1 \mathrm{~kg}($ S.D. $=12.7)$, but this was highly correlated with individual weight prior to infection $(r=0.62)$. Infected individuals lost on average $6 \%$ of their pre-infection weight.

\subsection{Haematology}

Results were received for the following parameters: red blood cells (RBC), white blood cells (WBC), haematocrit $(\mathrm{Ht})$, haemoglobin $(\mathrm{Hb})$, mean cell volume (MCV), mean cell 
haemoglobin $(\mathrm{MCH})$, mean cell haemoglobin concentration $(\mathrm{MCHC})$, platelets $(\mathrm{Pl})$, lymphocyte count, lymphocyte percentage (\%). Statistically significant results could be shown for the majority of parameters at the $95 \%$ confidence level when using the Fisher LSD method. There was a significant decline in RBC and lymphocyte counts, as well as $\mathrm{Ht}$ and lymphocyte percentages during the outbreak. The MCV and $\mathrm{MCH}$ values on the other hand were significantly higher during the FMD outbreak.

\subsection{Phylogenetic relationship of the virus obtained during the outbreak and further elucidation of the relationships of KNP buffalo isolates}

Previously most 1D gene sequence data was generated from buffalo in the south of the $\mathrm{KNP}$ as this was the region where culling occurred due to the higher density of buffalo and the close proximity to the abattoir (Bastos et al., 2001). Since the advent of sampling in the north of the KNP for BTB surveillance, it provided an opportunity for expanding the FMD sequence database. Buffalo isolates obtained between 1985 and 2005 as well as a single impala isolate from 1981 were included (Table 3). The KNP was arbitrarily divided into two geographical regions, namely northern and southern KNP (north and south of the Olifants river, respectively) (Fig. 1). The consensus tree is indicated in Fig. 3. 


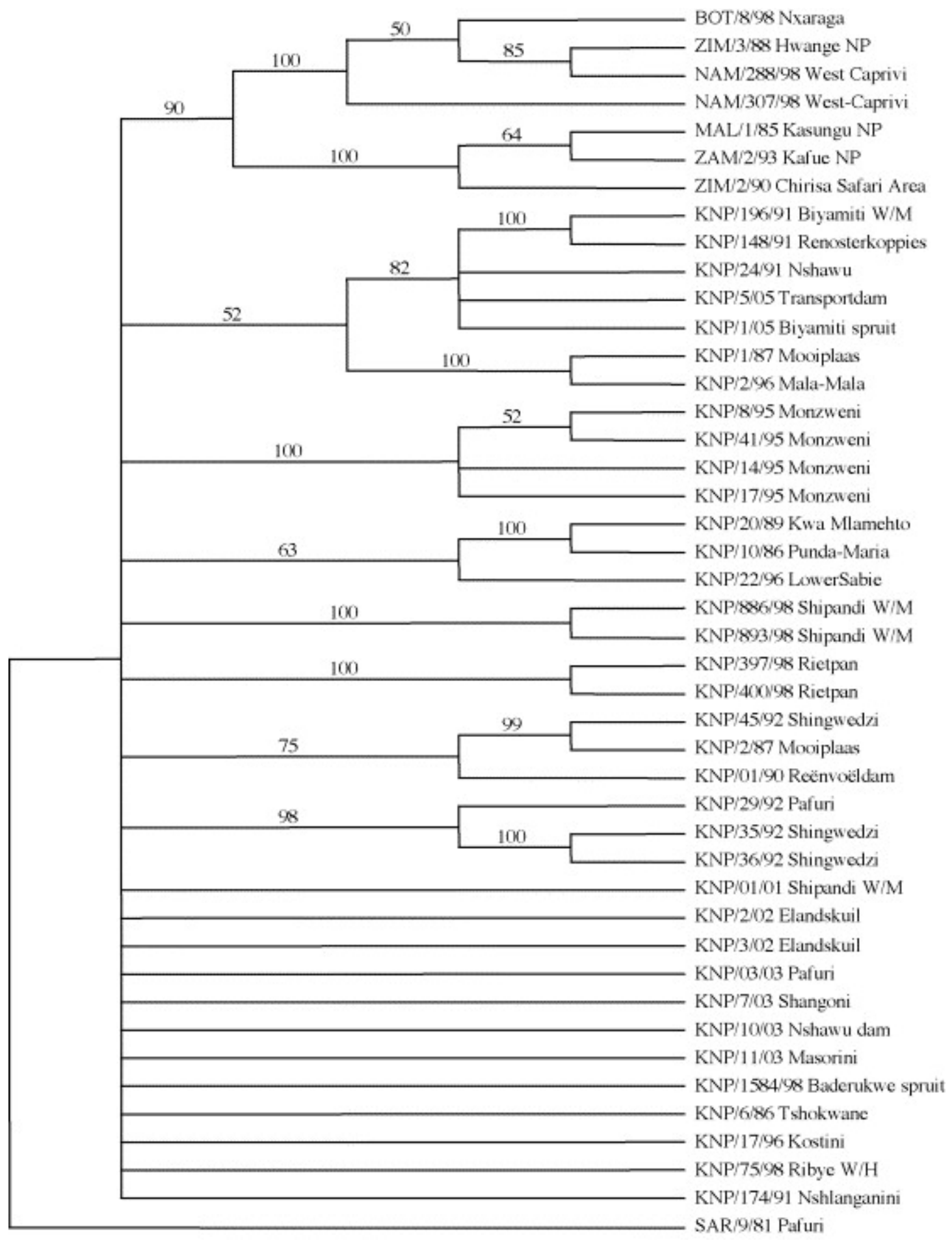

Fig. 3. Consensus phylogeny of the outbreak and related sequences as listed in Table 3. The nucleotide substitution model was HKY85 + invariants + rate heterogeneity with maximum likelihood estimates of the transition: transversion ratio, the proportion of 
invariant sites, and the parameter governing the shape of the gamma distribution being $3.55,0.31$, and 0.73 , respectively. Bootstrap values (estimated from 1000 neighborjoining trees based on the maximum likelihood model) are indicated on the tree.

All the buffalo isolates from the KNP included in this study grouped within topotype 1, earlier described to contain viruses from the KNP, south-eastern Zimbabwe and Mozambique (Fig. 3). The viruses from Botswana, Namibia, and western Zimbabwe grouped in topotype 2 and those from Malawi, Zambia, and northern Zimbabwe in topotype 3 (Bastos et al., 2001). The buffalo isolates from the outbreak described in this paper were all closely related to each other and only the first isolate, $\mathrm{KNP} / 01 / 01$, was included in the phylogenetic tree (Fig. 3).

The buffalo (LM11/z) from which this isolate was obtained was caught in 2000 at the Shipande Windmill (Fig. 1). This virus was not closely related to other isolates obtained from the same area (KNP/886/98 and KNP/893/98). No isolates were available from freeliving buffalo at the other localities (Malahlapanga, Klopperfontein, Tsombeyeni and Gadzingwe) where buffalo were caught for the BTB experiment.

Several examples were found where isolates obtained from the same herd on the same day were closely related for example $\mathrm{KNP} / 886 / 98$ and $\mathrm{KNP} / 893 / 98$ isolated at the Shipande windmill in 1998 (100\% bootstrap support), four isolates that were obtained during 1995 from Monzweni (KNP/8/95, KNP/14/95; KNP/17/95 and KNP/41/95-100\% support) and KNP/35/92 and KNP/36/92 from Shingwedzi in 1992 (Fig. 1 and Fig. 3). The opposite was also true where isolates obtained from the same herd on the same day demonstrated significant differences. KNP/45/92 also from Shingwedzi differed from $\mathrm{KNP} / 35 / 92$ and $\mathrm{KNP} / 36 / 92$ while $\mathrm{KNP} / 1 / 87$ and $\mathrm{KNP} / 2 / 87$ from Mooiplaas also demonstrated significant differences (Fig. 3). Viral sequences from the KNP are poorly resolved and it is not possible to identify the source of the virus within the park with any precision.

\subsection{Genetic variation in FMD virus isolates over 167 days}

Isolates obtained from animal LM7/z, LM11/z and LM15/z were chosen for further genetic studies as viruses could be obtained from these animals most frequently (Table 1). Isolates with the lowest passage level possible were chosen, as PCR directly from the 
probangs did not yield suitable sequence data. Nucleotide changes occurred in 15 positions (results not shown), leading to seven amino acid changes (Fig. 4). Three of these changes were evident in KNP/38/01 and KNP/33/01 (isolated on Days 159 and 176 from buffalo LM11/z, respectively): $\mathrm{G}$ to $\mathrm{S}$ at VP1 amino acid position 161; E to $\mathrm{V}$ at VP1 amino acid position 179; and V to M at VP1 amino acid position 24. Similarly $\mathrm{KNP} / 24 / 01$ and KNP/35/01 (isolated on Days 122 and 176 from buffalo LM15/z, respectively) had $\mathrm{D}$ while all other isolates had $\mathrm{G}$ at position $205 \mathrm{KNP} / 23 / 01$ isolated from LM11/z had two changes: $\mathrm{K}$ to $\mathrm{E}$ at VP1 amino acid position 44, and $\mathrm{E}$ to $\mathrm{K}$ at position 179. KNP/36/01 had Q in place of $\mathrm{H}$ at position 200. The RGD cell adhesion site was conserved in all isolates and 4 of the changes occurred in hypervariable sites previously described for SAT-1 (134-168 corresponding to the G-H loop, 173-186 corresponding to the H-I loop and 201-221 the C-terminus region of VP1 (Bastos et al., 2001 and Vosloo et al., 2006). 
openUP (July 2007)

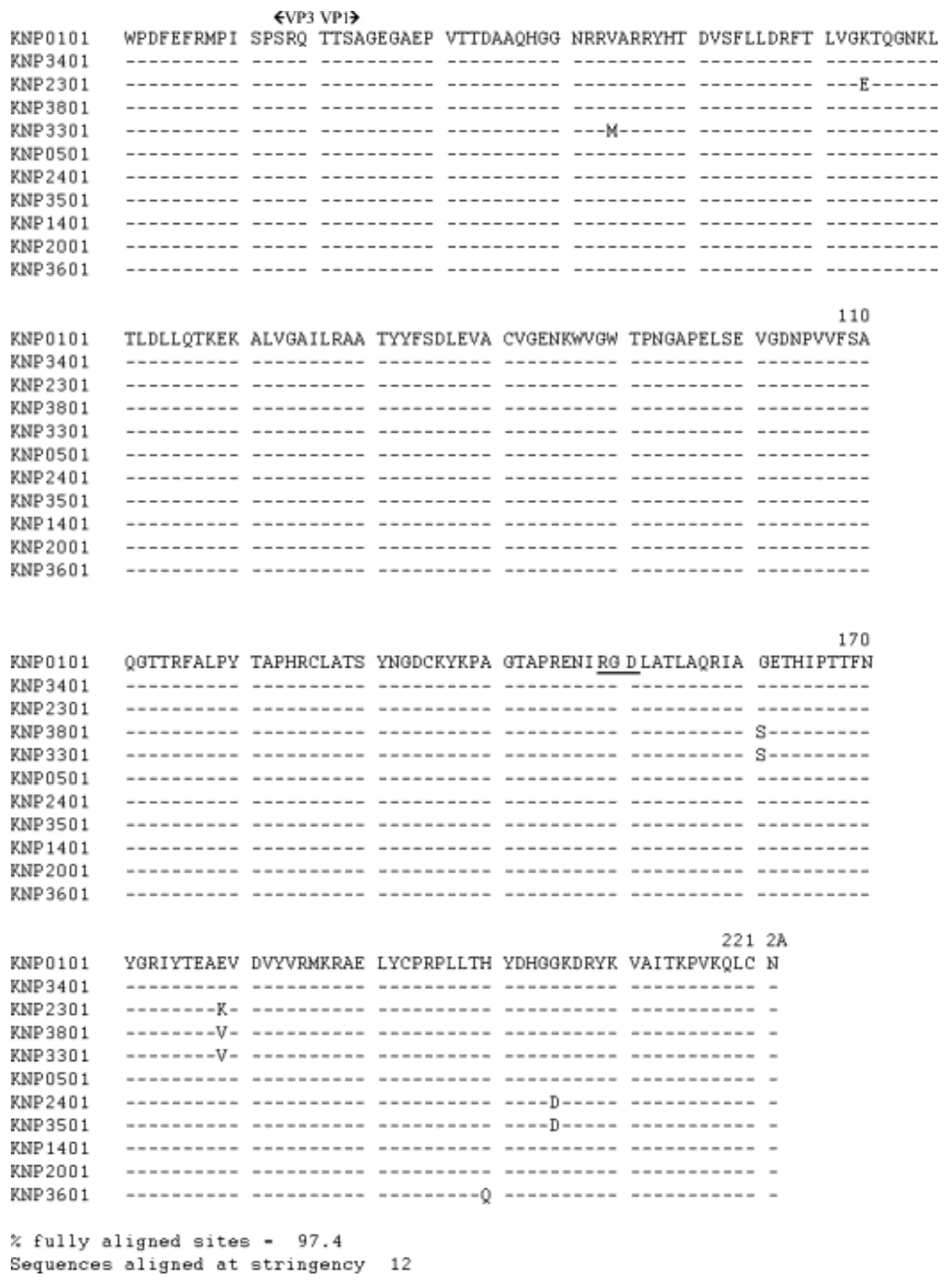

Fig. 4. Amino acid sequences inferred from 1D nucleotide data.

The TCS genealogy (Fig. 5) demonstrates that the genetic data is consistent with the epidemiological data indicating that infection arose first in LM11/z and spread from this individual directly to the others for which sequence data is available. Nucleotide 
substitutions accumulated over time, with five different substitutions arising in LM15/z, and as many as nine in LM11/z. The rate of the molecular clock is estimated to be $3 \times 10^{-5}$ nucleotide substitutions per site per day $\left(95 \%\right.$ confidence intervals $8 \times 10^{-6}$ to $\left.5.7 \times 10^{-5}\right)$.

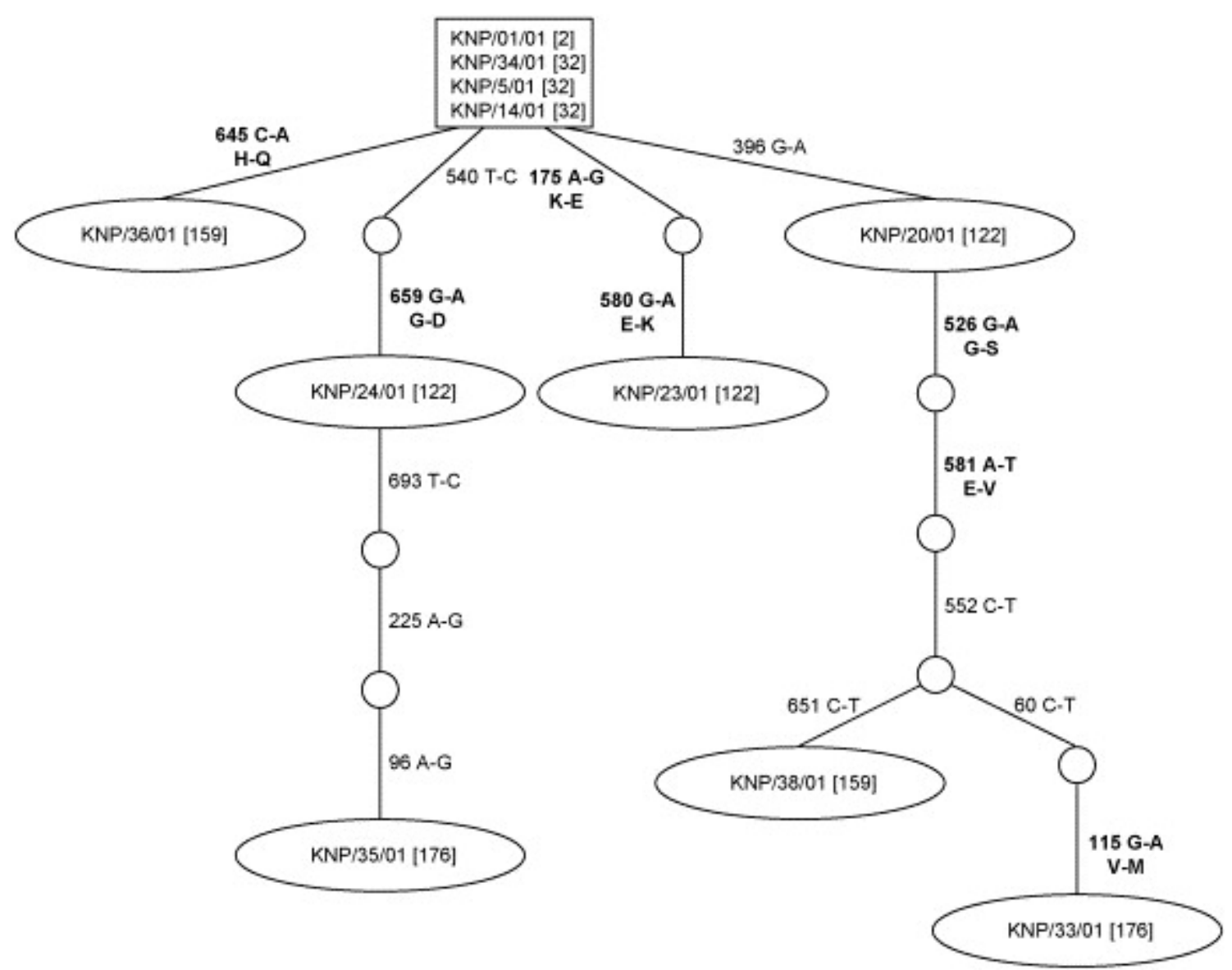

Fig. 5. TCS diagram indicating the genealogy of the sequences obtained from infected buffalo. The square box indicates the index sequence, and circles indicate unsampled "ancestral" sequences. The position and type of nucleotide substitutions consistent with each branch are indicated adjacent to each branch, and the date of virus isolated (measured in number of days since the start of the outbreak) is indicated in square brackets. Nucleotide substitutions indicated in bold are non-synonymous, and the subsequent amino acid changes indicated below the denoted nucleotide substitution. 


\section{Discussion}

Buffalo are important for their role in maintaining FMD infection and their ability of infecting other susceptible species in sub-Saharan Africa. They have been shown to be the source of infection for impala and domestic animals in proximity of the KNP and other game parks in southern Africa. Furthermore, it has been shown experimentally that buffalo can transmit virus to susceptible cattle (Thomson and Bastos, 2004 and Vosloo and Thomson, 2004). For this reason extreme care is taken in South Africa to separate wildlife and domestic animals where fences are a mainstay in the success of the country in maintaining a zone of FMD freedom without vaccination.

Clinical FMD is rare in free-living buffalo and hardly ever documented. Between 1970 and 1971 when FMD caused by SAT-2 was abundant in free-living wildlife in the KNP, $1.7 \%$ of buffalo carcasses showed lesions suggestive of FMD. This prevalence varied from 25 to $34 \%$ in small random groups. However, confirmation by virus isolation was not possible due to the small size of the lesions (Young et al., 1972) and it is therefore possible that these lesions were not due to FMD. During this time, a small group of captive buffalo became infected with SAT-2 demonstrating similar signs to what was observed in the outbreak described in this paper. All the captive buffalo were seronegative and only one animal had antibodies to all three serotypes, and was previously shown to carry a SAT-3 virus (Young et al., 1972). The seven other animals were therefore fully susceptible and they showed clinical signs in contrast to the animal that had antibodies to SAT-2 and was protected. It is also possible that disease may have spread from this animal to its cohorts, as sophisticated methods such as nucleotide sequencing were not available at the time to determine the exact origin of the disease. In contrast to the outbreak described here, animals during the previous disease incidence showed a painful gait with obvious feet lesions and salivation (Young et al., 1972). Clinical disease depends on the level of immunity, virus strain, strains with a predilection for certain species, quantity of virus the animals are exposed to, host species, breed and differences can even occur between individuals of a breed (Thomson and Bastos, 2004). It is therefore not surprising that the disease symptoms differed between these two outbreaks in captive buffalo. Firstly, the outbreaks were caused by different serotypes, and during the SAT-1 outbreak none of the animals were naïve as all had tested positive 
for antibodies to all three serotypes by the time they were captured (results not shown). At the time of the outbreak described in this paper, there was no record of FMD outbreaks in other species in the KNP supporting the suggestion that the outbreak was caused by carrier animals within the group. Unfortunately the phylogenetic tree was not sufficiently resolved to support or refute this theory. Buffalo can move significant distances in the KNP during drought or other ecological pressures and single bulls as well as subsets of herds have been shown to move between herds (Funston et al., 1994 and Mloszewski, 1983). This could explain why the tree did not resolve into geographically linked clusters of FMD viruses. Unless very closely related viruses are compared, it will not be possible to trace the origin of buffalo in the KNP.

It is still not clear how disease spreads from carrier buffalo to in contact animals of the same and different species. For buffalo calves it is postulated that disease spreads like a childhood epidemic when maternal antibodies wane nearly at the same time in these seasonal breeders, leaving a fully susceptible sub-adult group (Thomson et al., 1992). During this outbreak of SAT-1 most of the animals had antibodies to all three serotypes and were therefore not naïve and could have been carriers of FMD virus. It is possible that the strain that caused the outbreak was significantly antigenically different to the ones animals had been exposed to previously to allow the virus to escape neutralisation and cause clinical disease. These animals were initially selected from five different herds, and it is clear from the phylogenetic analysis that within the northern region of the KNP, significantly different viruses occur.

In addition it has been suggested that sexual transmission is possible (Bastos et al., 1999). Buffalo heifers reach sexual maturity between 2 and 3 years of age, whereas spermatogenesis commences in buffalo bulls at 2.5 years of age. In the KNP the buffalo breeding season runs from December to May. The index case was a 30-month-old heifer that became infected in May, and most of the bulls in the experiment varied between 14 months and 2.5 years of age. It could therefore be speculated that sexual transmission between buffalo bulls and a heifer occurred. However, none of the animals were pregnant at the time of slaughter, five months later.

Alternatively the stress of captivity, frequent immobilisation for sampling and BCG vaccination may have led to the spread of the virus. Although circumstantial evidence has 
pointed to stress being a possible reason for virus transmission from carrier buffalo to susceptible cattle in Zimbabwe (Hedger and Condy, 1985), stress induced in carrier cattle using dexamethasone refuted this theory (Ilott et al., 1997). Similarly co-infection with rinderpest and bovine herpes 1 viruses, respectively, did not increase the level of virus recovery in carrier cattle or transmission (McVicar, 1977). There were no significant differences between any of the parameters tested in this study, between animals vaccinated with $\mathrm{BCG}$ and the unvaccinated controls.

In the KNP, buffalo calves first become infected by SAT-1, followed by SAT-2 and lastly SAT-3 based on serological evidence (Thomson and Bastos, 2004). SAT-1 viruses have also been recovered from persistently infected buffalo at a higher rate (Thomson et al., 2003). In contrast most outbreaks in the southern African region are caused by SAT-2 followed by SAT-1 and lastly SAT-3 which has led to speculation that SAT-2 is better adapted for spread to other species such as cattle and impala (Thomson and Bastos, 2004), and SAT-1 may be better adapted for spread among buffalo. However, there is no experimental evidence to date to support these assumptions.

In cattle the virus levels in the oesophageo-pharyngeal region begin to decline soon after infection (Burrows, 1966 and Donaldson et al., 1981) and the levels may fluctuate with virus detection becoming erratic by six months post infection (Gebauer et al., 1988 and Van Bekkum et al., 1959). In excess of 50\% of cattle that had recovered from infection become persistently infected (Kitching, 2002). Persistence occurs in the face of an antibody response in the oesophageo-pharyngeal secretions and super-infection with more than one type can occur (McVicar and Sutmoller, 1976 and Van Bekkum et al., 1959). Virus isolation in the infected buffalo also became less successful with nearly $70 \%$ of the animals positive one and four months post infection, decreasing to $40 \%$ at five months and virus could be isolated from only $17 \%$ of the animals after six months, when the experiment was terminated.

The weight loss observed during the outbreak was due to FMD infection and not other reasons, as the animals were regularly treated for internal parasites. Anorexia was also observed during the 1971 outbreak (Young et al., 1972). Maximum weight loss during outbreaks was shown here to be proportional the pre-outbreak weights of individuals. 
The buffalo presented with a significant lymphopenia that lasted approximately 100 days. Cattle infected with FMD similarly showed a decrease in total T-cells, affecting both T4 and CD8 subsets (Sigal et al., 1992). The cattle were only monitored one week post infection, therefore a correlation between the recovery of total lymphocytes in cattle and that for buffalo found in this paper cannot be made. Furthermore, the subsets of cells affected in buffalo were not investigated. In acutely infected pigs the total number of lymphocytes demonstrated a significant lymphopenia that peaked at 2 days post infection, began to recover at 4 days and was resolved by day 5 (Bautista et al., 2003), a significant difference with the buffalo. This period correlated with the time of viremia in infected pigs. Since no virus could be isolated from washed PBMC at the peak of viremia, the loss of T-cells was not due to infection of these cells. Bautista et al. (2003) showed similar results when lymphocytes were counted as a percentage of all cells, but when tracking total white blood cell counts, there was only a mild leukopenia on Day 3, with no statistically significant difference with controls. When using another strain of virus, the lymphopenia in infected pigs were milder, suggesting that the level of lymphopenia correlates to the virulence of the infecting virus as measured by clinical outcome. All subsets of T-cells were affected during FMD infection resulting in a transient immunosuppressed state in infected animals. During this time the ability of circulating Tcells to respond to mitogen was also negatively influenced (Bautista et al., 2003). SAT type viruses undergo high levels of mutation over time in persistently infected buffalo and these changes can lead to significant antigenic changes (Vosloo et al., 1996). A daily rate of 0.00003 nucleotides per site per day, would lead to a $1 \%$ turnover in capsid nucleotides per year-which is well in the $0.5-1.5 \%$ per year range previously reported (Haydon et al., 2001 and Vosloo et al., 1996). In this case, almost half these substitutions resulted in amino acid changes, so antigenic change is likely. Such high rates of change suggest that it may be possible to trace individual transmission events between animals using sequence data.

The TCS genealogy demonstrated that viruses obtained from animal LM11/z on Days 159 and 176 shared a mutation at position 396 with sequence KNP/20/01 derived from LM7/z. Possible explanations for this are that LM7/z superinfected LM11/z later on in the epidemic, spreading mutation G-A at position 396 from LM7/z to LM11/z, and the 
virus then continued to incur mutations at 526,581, 552, 651, 60, and 115; alternatively mutation G-A 396 arose in two animals independently; once in LM11/z after sampling $\mathrm{KNP} / 23 / 01$ but before sampling KNP/38/01 and KNP33/01, and once in LM7/z prior to Day 122.

\section{Conclusion}

The clinical manifestation of FMD in African buffalo has only been described once before. However, the weight loss and significant and long-lasting lymphopenia have not been observed previously. The rate of change of FMD virus within these buffalo has indicated again how important buffalo are for the generation of variants of viruses, emphasising their significant role in the epidemiology of FMD and its control in southern Africa.

\section{References}

Bastos et al., 2001 A.D.S. Bastos, D.T. Haydon, R. Forsberg, N.J. Knowles, E.C. Anderson, R.G. Bengis, L.H. Nel and G.R. Thomson, Genetic heterogeneity of SAT-1 type foot-and-mouth disease viruses in southern Africa, Arch. Virol. 146 (2001), pp. 1537-1551.

Bastos et al., 2000 A.D.S. Bastos, C.I. Boshoff, D.F. Keet, R.G. Bengis and G.R. Thomson, Natural transmission of foot-and-mouth disease virus between African buffalo (Syncerus caffer) and impala (Aepyceros melampus) in the Kruger National Park, S. Afr. Epid. Infect. 124 (2000), pp. 591-598.

Bastos, 1998 A.D.S. Bastos, Detection and characterisation of foot-and-mouth disease virus in sub-Saharan Africa, Onderstepoort J. Vet. Res. 65 (1998), pp. 37-47.

Bastos et al., 1999 A.D.S. Bastos, H.J. Bertschinger, C. Cordel, C. Van Vuuren, W. De, D. Keet, R.G. Bengis, D.G. Grobler and G.R. Thomson, Possibility of sexual transmission of foot-and-mouth disease from African buffalo to cattle, Vet. Rec. 145 (1999), pp. 77-79. 
Bautista et al., 2003 E.M. Bautista, G.S. Ferman and W.T. Golde, Induction of lymphopenia and inhibition of $\mathrm{T}$ cell function during acute infection of swine with footand-mouth disease virus (FMDV), Vet. Immunol. Immunopathol. 92 (2003), pp. 61-73. Beck and Strohmaier, 1987 E. Beck and K. Strohmaier, Subtyping of European foot-andmouth disease virus strains by nucleotide sequence determination, J. Virol. 61 (1987) (5), pp. 1621-1629.

Boom et al., 1990 R. Boom, I.C.J. So, M.M.M. Salimans, C.L. Jansen, P.M.E. WertheimVan Dillen and J. Van Der Noordaa, Rapid and simple method for purification of nucleic acids, J. Clin. Microbiol. 28 (1990), pp. 495-503.

Brückner et al., 2002 G.K. Brückner, W. Vosloo, B.J.A. Du Plessis, P.E.L.G. Kloeck, L. Connoway, M.D. Ekron, D.B. Weaver, C.J. Dickason, F.J. Schreuder, T. Marais and M.E. Mogajane, Foot-and-mouth disease: the experience in South Africa, Rev. Sci. Tech. OIE 21 (2002) (3), pp. 751-764.

Burrows, 1966 R. Burrows, Studies on the carrier state of cattle exposed to foot-andmouth disease virus, J. Hyg. 64 (1966), pp. 81-90.

Clement et al., 2000 M. Clement, D. Posada and K. Crandall, TCS: a computer program to estimate gene genealogies, Mol. Ecol. 9 (2000), pp. 1657-1660.

Donaldson et al., 1981 A.I. Donaldson, A.J.M. Garland, N.P. Ferris and T. Collen, The 1975 foot-and-mouth disease epidemic in Malta. I. Experimental studies with the causal virus strain O1 Malta, Br. Vet. J. 137 (1981), pp. 300-307.

Drummond et al., 2002 A.J. Drummond, G.K. Nicholls, A.G. Rodrigo and W. Solomon, Estimating mutation parameters, population history and genealogy simultaneously from temporally spaced sequence data, Genetics 161 (2002), pp. 1307-1320.

Funston et al., 1994 P.J. Funston, J.D. Skinner and D.M. Dott, Seasonal variation in movement patterns, home range and habitat selection of buffaloes in a semi-arid habitat, Afr. J. Ecol. 32 (1994), pp. 100-114.

Gebauer et al., 1988 F. Gebauer, J.C. De la Torre, I. Gomes, M.G. Maten, H. Barahona, B. Tiraboshi, I. Bergman, P.A. De Mello and E. Domingo, Rapid selection of genetic and antigenic variants of foot-and-mouth disease during persistence in cattle, Virology 62 (1988), pp. 2041-2049. 
Hargreaves et al., 2004 S.K. Hargreaves, C.M. Foggin, E.C. Anderson, A.D.S. Bastos, G.R. Thomson, N. Ferris and N. Knowles, An investigation into the source and spread of foot-and-mouth disease virus from a wildlife conservancy in Zimbabwe, Rev. Sci. Tech. 23 (2004) (3), pp. 783-790.

Harley, 2001 E.H. Harley, DAPSA. DNA and protein sequence analysis, version 4.91, Department of Chemical Pathology, University of Cape Town, South Africa (2001). Haydon et al., 2001 D.T. Haydon, A.R. Samuel and N.J. Knowles, The generation and persistence of genetic variation in foot-and-mouth disease virus, Prev. Vet. Med. 51 (2001), pp. 111-124.

Hedger and Condy, 1985 R.S. Hedger and J.B. Condy, Transmission of foot-and-mouth disease from African buffalo virus carriers to bovines, Vet. Rec. 117 (1985), p. 205. Hedger et al., 1972 R.S. Hedger, J.B. Condy and S.M. Golding, Infection of some species of African wildlife with foot-and-mouth disease virus, J. Comp. Pathol. 82 (1972), pp. $455-461$.

Ilott et al., 1997 M.C. Ilott, J.S. Salt, R.M. Gaskell and R.P. Kitching, Dexamethasone inhibits virus production and the secretory IgA response in the oesophageal-pharyngeal fluid in cattle persistently infected with foot-and-mouth disease virus, Epidemiol. Infect. 118 (1997), pp. 181-187.

Kitching, 2002 R.P. Kitching, Clinical variation in foot-and-mouth disease: cattle, Rev. Sci. Tech. 21 (2002), pp. 499-504.

McVicar and Sutmoller, 1976 F.W. McVicar and P. Sutmoller, Growth of foot-andmouth disease virus in the upper respiratory tract of non-immunized, vaccinated, and recovered cattle after intranasal inoculation, J. Hyg. 76 (1976), pp. 467-481.

McVicar, 1977 J.W. McVicar, The pathobiology of foot-and-mouth disease in cattle: a review, Bull Centro Panamericano Fiebre Aftosa 26 (1977), pp. 9-14.

Mloszewski, 1983 M.J. Mloszewski, The Behaviour and Ecology of the African Buffalo (first ed.), Cambridge University Press, Cambridge (1983), pp. 63-137.

Sigal et al., 1992 L.J. Sigal, G. Gomez and M. Braun, Changes in mononuclear peripheral blood cells in cattle with foot-and-mouth disease, Vet. Immunol. Immunopathol. 30 (1992), pp. 431-438. 
Sutmoller and Gaggero, 1965 P. Sutmoller and A. Gaggero, Foot-and-mouth diseases carriers, Vet. Rec. 77 (1965) (33), pp. 968-969.

Swofford, 2002 Swofford, D.L., 2002. PAUP* 4.0b10. Sinauer Associates, Sunderland, MA.

Thomson et al., 2003 G.R. Thomson, W. Vosloo and A.D. Bastos, Foot-and-mouth disease in wildlife, Virus Res. 91 (2003), pp. 145-161.

Thomson et al., 1992 G.R. Thomson, W. Vosloo, J.J. Esterhuysen and R.G. Bengis, Maintenance of foot-and-mouth disease viruses in buffalo (Syncerus caffer Sparrman, 1779) in southern Africa, Rev. Sci. Tech. 11 (1992), pp. 1097-1107.

Thomson and Bastos, 2004 G.R. Thomson and A.D.S. Bastos In: J.A.W. Coetzer and R.C. Tustin, Editors, Foot-and-Mouth Disease. Infectious Diseases of Livestock (second ed.), Oxford University Press, South Africa (2004), pp. 1324-1365.

Van Bekkum et al., 1959 J.G. Van Bekkum, H.S. Frenkel, H.H.J. Frederiks and S. Frenkel, Observations on the carrier state of cattle exposed to foot-and-mouth disease virus, Tijdschrift voor Dieregeneeskunde 84 (1959), pp. 1159-1164.

Vosloo and Thomson, 2004 W. Vosloo and G.R. Thomson, Natural Habitats in which Foot-and-Mouth Disease Viruses are Maintained. In: E. Domingo and F. Sobrino, Editors, Foot-and-Mouth Disease: Current Perspectives, Horizon Bioscience, Great Britain (2004), pp. 383-410.

Vosloo et al., 2002 W. Vosloo, A.D.S. Bastos, O. Sangare, S.K. Hargreaves and G.R. Thomson, Review of the status and control of foot-and-mouth disease in sub-Saharan Africa, Rev. Sci. Tech. 21 (2002), pp. 437-449.

Vosloo et al., 1996 W. Vosloo, A.D. Bastos, E. Kirkbride, J.J. Esterhuysen, D. Janse van Rensburg, R.G. Bengis, D.F. Keet and G.R. Thomson, Persistent infection of African buffalo (Syncerus caffer) with SAT-type foot-and-mouth disease viruses: Rate of fixation of mutations, antigenic change and interspecies transmission, J. Gen. Virol. 77 (1996), pp. 1457-1467.

Vosloo et al., 2006 W. Vosloo, A.D.S. Bastos and C.I. Boshoff, Retrospective genetic analysis of SAT-1 type foot-and-mouth disease outbreaks in southern Africa, Arch. Virol. 151 (2006), pp. 285-289. 
Young et al., 1972 E. Young, R.S. Hedger and P.G. Howell, Clinical foot-and-mouth disease in the African buffalo (Syncerus caffer), Onderstepoort J. Vet. Res. 39 (1972) (3), pp. 181-184.

Corresponding author at: Onderstepoort Veterinary Institute, Private Bag X05, Onderstepoort 0110, South Africa. Tel.: +27 12 5299592; fax: +27 125299595 . 